Bài báo khoa học

\title{
Áp dụng thử nghiệm phần mềm mã nguồn mở OpenFOAM mô phỏng lũ bùn đá
}

\author{
Đặng Trường An ${ }^{1^{*}}$ \\ ${ }^{1}$ Trường Đại học Khoa học Tự Nhiên, Đại học Quốc gia TP. HCM; dtan@hcmus.edu.vn \\ *Tác giả liên hệ: dtan@hcmus.edu.vn; Tel.: +84-909719878 \\ Ban Biên tập nhận bài: 12/3/2021; Ngày phản biện xong: 9/5/2021; Ngày đăng bài: \\ $25 / 6 / 2021$
}

Tóm tắt: Lũ bùn đá là một dạng dòng chảy năng lượng cao chứa nước, đất, cát và sỏi đá trộn lẫn với nhau thường hình thành ở các vùng đồi núi dốc. Năng lượng mà lũ bùn đá tạo ra khi va chạm với các chướng ngại vật trên đường di chuyển của chúng thường rất lớn, có thể gây thiệt hại về người và tài sản. Trong nghiên cứu này, phần mềm OpenFOAM bước đầu được áp dụng thử nghiệm để mô phỏng dòng lũ bùn đá của một kênh thực nghiệm. Khả năng mô phỏng lũ bùn đá của phần mềm được đánh giá thông qua so sánh các chỉ số thống kê sai số giữa kết quả mô phỏng của nó với dữ liệu đo đạc và với kết quả mô phỏng từ phần mềm InterMixingflow. Sai số $\mathrm{R}_{\text {mean }}$ và RMSE giữa kết quả mô phỏng của phần mềm với dữ liệu thực đo lần lượt là 0,72 và 5,43 trong khi giá trị tương ứng từ phần mềm InterMixingflow là 0,85 và 5,83 . Thông qua phân tích các chỉ số sai số từ phần mềm đã áp dụng và phần mềm InterMixingflow, có thể thấy phấn mềm OpenFOAM rất có triển vọng để xem xét áp dụng mô phỏng lũ bùn đá cho các trường hợp kênh tự nhiên.

Từ khóa: Lũ bùn đá; OpenFOAM; Áp suất cao; Bão; Dòng năng lượng cao.

\section{Mở đầu}

Lũ bùn đá là một hiện tượng tự nhiên thường xuất hiện ở các vùng đồi núi, năng lượng được tạo ra khi dòng chảy này tiếp xúc với vật cản trên đường chúng di chuyển là rất lớn [13]. Theo [4] dòng chảy lũ bùn là một dang dòng chảy bao gồm hỗn hợp nước, đất, đá và thậm chí chứa cả vật liệu thô xảy ra phổ biến ở khu vực đồi núi gây thiệt hại lớn về người và tài sản. Do dòng chảy lũ bùn pha trộn nhiều loại vật liệu khác nhau nên năng lượng chúng tạo ra lớn hơn nhiều so với dòng chảy chỉ chứa nước và vì thế thiệt hại do chúng gây ra cho các khu vực chịu tác động trực tiếp là rất lớn $[3,5-7]$. Cho đến này, các khảo sát thực địa để tìm hiểu nghiên cứu về dòng chảy lũ bùn vẫn còn gặp phải nhiều thách thức do chi phí khá tốn kém và mất an toàn đối với người tham gia khảo sát [7-9]. Chính vì thế, các tiếp cận nghiên cứu về dòng chảy lũ bùn đang được áp dụng phổ biến hiện nay là sử dụng thiết bị công nghệ bay độ phân giải cao giám sát bề mặt (Airborne Lidar) [10-12]. [10] đã tiến hành một nghiên cứu về lũ bùn đá xảy ra tại thị trấn Matata ở Plenty, phía Đông New Zealand. Nhóm nghiên cứu đã sữ dụng thiết bị bay độ phân giải cao Airborne Lidar để giám sát bề mặt đất của khu vực xảy ra lũ bùn sau sự kiện mưa cực lớn. Sự kiện mưa này gây ra hiện tượng sạt trượt đất, đá trên diện rộng và dòng chảy lũ bùn gây ảnh hưởng lớn đến khu vực Awatarariki và Waitepuru thuộc Matata. Nghiên cứu đã chỉ ra, việc áp dụng công nghệ bay giám sát Airborne Lidar nghiên cứu dòng chảy lũ bùn cho thấy triển vọng khả quan của kỹ thuật này. Năm 2016, [12] đã áp dụng thiết bị công nghệ bay Airborne Lidar giám sát những thay đổi về bề mặt địa hình do dòng chảy lũ bùn gây ra cho khu vực Umyeon, Hàn Quốc. Trong nghiên cứu đã sử 
dụng thiết bị Airborne Lidar bay giám sát xung quanh khu vực xảy ra lũ bùn và rút ra nhận xét rằng, phương pháp sử dụng công nghệ bay Airborne Lidar trong giám sát dòng chảy lũ bùn nên được khuyến khích áp dụng nhiều hơn cho các khu vực thường xảy ra lũ bùn khác của Hàn Quốc cũng như các khu vực trên thế giới. Bên cạnh sử dụng công nghệ bay Airborne Lidar để giám sát, nghiên cứu về dòng chảy lũ bùn, hướng tiếp cận sử dụng mô hình số mô phỏng diễn biến dòng chảy lũ bùn cũng được áp dụng rộng rãi ở nhiều nơi trên thế giới [1315]. Cụ thể, năm 2013 [16] đã tiến hành nghiên cứu đánh giá khả năng áp dụng của mô hình số mô phỏng dòng chảy lũ bùn cho 16 trường hợp kênh thực nghiệm được thiết lập tại Viện Kỹ thuật Giám sát Rủi ro Miền núi thuộc trường Đại học Tài nguyên Thiên nhiên và Đời sống Vienna.

Kết quả nghiên cứu chỉ ra rằng, phương pháp tiếp cận mô hình số mô phỏng dòng chảy lũ bùn đá cho sự tương đồng tốt với dữ liệu đo đạc thực nghiệm từ mô hình vật lý phòng thí nghiệm. Một nghiên cứu được tiến hành bởi [17] sử dụng mô hình số 3 chiều Mohr-Coulomb dựa trên 2 pha dòng chảy rắn (bùn, đá) và lỏng (nước) cho kênh thực nghiệm có độ dốc lớn. Các kết quả mô phỏng chỉ ra khả năng ứng dụng tiềm năng của mô hình số trong mô phỏng các quá trình thủy động học phức tạp của các dòng chảy hỗn hợp lũ bùn với vật liệu rắn có kích thước hạt khá đa dạng. Việc khảo sát thực địa để nghiên cứu về dòng chảy lũ bùn như được trình bày còn đối mặt nhiều thách thức và chưa được tiến hành rộng rải, trong khi tiếp cận dựa vào thiết bị công nghệ bay Airborne Lidar còn khá tốn kém thì mô hình số được xem là hướng tiếp cận dễ khả thi hiện nay [1,13-14].

Chính vì vậy, mục tiêu của nghiên cứu này là: a) áp dụng phần mềm mã nguồn mở OpenFOAM mô phỏng dòng chảy hỗn hợp lũ bùn cho trường hợp kênh nhân tạo được xây dựng từ phòng thí nghiệm và b) tiến tới xem xét áp dụng phần mềm mô phỏng dòng chảy lũ bùn cho trường hợp kênh tự nhiên trong một nghiên cứu tiếp theo.

\section{Phương pháp nghiên cứu và dữ liệu}

\subsection{Khu vục nghiên cứu}

Trong nghiên cứu này, dữ liệu để tiến chạy mô phỏng lũ bùn đá được thu thập từ [8]. Kênh thực nghiệm xây dựng trong phòng thí nghiệm dựa trên hình dạng lũ bùn xảy ra trong kênh tự nhiên (Hình $1 \mathrm{a}$ ) và kênh thực nghiệm trong trường hợp nghiên cứu này được thiết kế có chiều dài 7,5 $\mathrm{m}$ và rộng $2,0 \mathrm{~m}$ (Hình $1 \mathrm{~b}$ ). Kênh thực nghiệm được chia 2 đoạn với độ dốc kênh thay đổi từ 10 đến $30^{\circ}$. Trong đó, đoạn kênh thứ $\mathrm{I}$ dài $4,4 \mathrm{~m}$ và độ dốc kênh $\left(\beta=30^{\circ}\right)$ và đoạn kênh thứ II có chiều dài $3,1 \mathrm{~m}$ với độ dốc $\left(\beta=10^{\circ}\right)$ (Hình $\left.1 \mathrm{~b}\right)$.

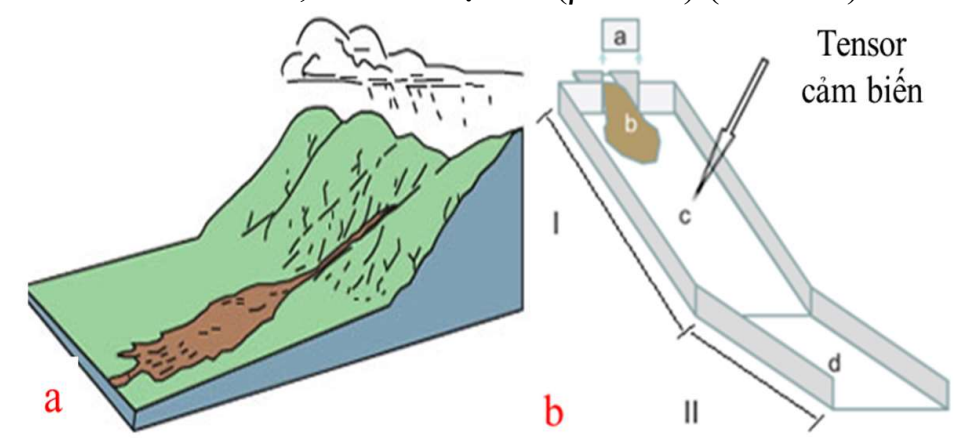

Hình 1. Phác thảo lũ bùn đá a) kênh tự nhiên và b) kênh thực nghiệm quy mô phòng thí nghiệm $[8,15]$.

\subsection{Tổng quan về phần mềm OpenFOAM}

OpenFOAM được viết tắc từ cụm từ-Open Source Field Operation And Manipulation, là phần mềm nguồn mở được phát triển bởi Đại học Imperial, Anh Quốc [19]. Phần mềm này được Henry Weller xây dựng dựa trên ý tưởng chính lấy từ mô phỏng động lực học cơ lưu 
chất (CFD-Computational Fluid Dynamics) với phương pháp thể tích hữu hạn được sử dụng làm thuật giải [19, 20-22]. Năm 2000, phần mềm OpenFOAM được công bố như một phần mềm thương mại [19]. Sau đó, phần mềm OpenFOAM được sở hữu bởi Quỹ OpenFOAM và đến những năm 2013, phần mềm này được hợp nhất cho cả hai phiên bản thương mại và mã nguồn mở [20-21]. Các module của phần mềm OpenFOAM viết trên nền tảng lập trình hướng đối tượng sử dụng ngôn ngữ $\mathrm{C}++[19]$. OpenFOAM là tập hợp của khoảng 250 chương trình con được nhúng trong hơn 100 module [19-22]. Về cơ bản, các chương trình con của phần mềm OpenFOAM được tổ chức thành 3 khối (Hình 2) bao gồm: (1) Khối tiền xử lý (Pre-processing) gồm các công cụ mô hình hóa và chia lưới; (2) Khối giải (Solving) gồm các bộ giải chuẩn được cung cấp sẵn hoặc do người dùng tự xây dựng và bổ sung thêm vào thư viện của OpenFOAM; và (3) Khối phân tích, thể hiện lời giải (Post-processing) gồm các thư viện công cụ phục vụ việc biểu diễn, hiển thị kết quả tính toán.

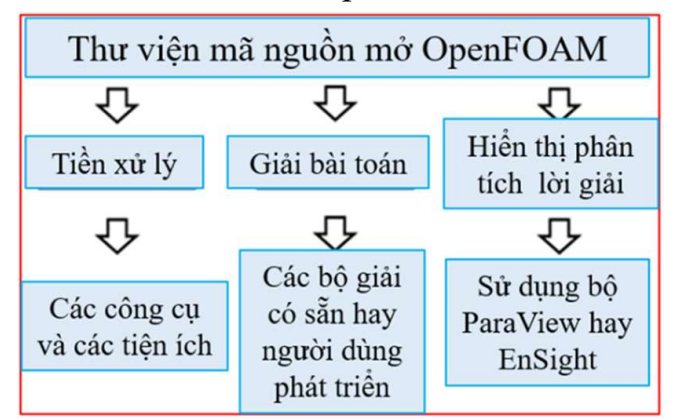

Hình 2. Phác thảo ba khối hoạt động trong phần mềm OpenFOAM.

\subsection{Co sở lý thuyết của phần mềm OpenFOAM}

Hầu hết các chương trình con mô phỏng dòng chảy trong phần mềm OpenFOAM sử dụng một trong các thuật toán PISO-Pressure-Implicit Split-Operator, SIMPLE-SemiImplicit Method for Pressure-Linked Equations hay kết hợp cả hai thuật giải PISO và SIMPLE được gọi với tên PIMPLE [20]. Trong đó, thuật giải PIMPLE thực thi vòng lặp ghép nối tích hợp phương trình động lượng và bảo toàn khối lượng từ các phương trình rối RANS (hệ phương trình Reynolds-averaged Navier-Stokes) [20, 23]. Thuật giải PIMPLE tích hợp trong phần mềm OpenFOAM có thể áp dụng cho các nghiên cứu:

Mô phỏng hoạt động của cánh turbine tàu ngầm, mô phỏng tương tác giữa thuyền và nước, mô phỏng diễn biển vỡ đập và mô phỏng chuyển động của dòng chảy lũ bùn. Phần mềm OpenFOAM được xây dựng dựa trên hệ phương trình chủ đạo sau:

Phương trình liên tục

Phương trình vận chuyển

$$
\nabla \cdot U=0
$$

$$
\frac{\partial \alpha_{m}}{\partial t}+\nabla \cdot\left(U \alpha_{m}\right)=0
$$

Phương trình động lượng

$$
\frac{\partial(\rho U)}{\partial t}+\nabla \cdot(\rho U \times U)=-\nabla p+\nabla \cdot \mathbf{T}+\rho f
$$

Trong đó $U$ là thành phần vận tốc; $\rho$ là mật độ trung bình của các chất; $\mathbf{T}$ là tensor ứng suất nhớt của hỗn hợp; f là tổng các lực khác; $\alpha_{m}$ là tổng của phân số biểu thị thành phần của thành phần bùn và cát tại một điểm không thời gian [19].

Trong đó $\alpha_{m}$ trong phương trình (2) được xác định bởi phương trình (4).

$$
\alpha_{m}=\alpha_{2}+\alpha_{3}
$$

Phần không khí cũng ảnh hưởng đến $\alpha_{m}$ này qua phương trình (5).

$$
\alpha_{1}=1-\alpha_{m}
$$


Trong đó $\alpha_{1}$ là tham số biểu thị thành phần không khí; $\alpha_{2}$ là tham số biểu thị thành phần bùn; $\alpha_{3}$ là tham số biểu thị thành phần cát.

Phương trình (2) chỉ xét đến pha lỏng, nên cần phải xét thêm pha khí cho các mô phỏng dòng chảy bùn. Đối với pha rắn (dòng chảy bùn) phương trình vận chuyển có dạng sau:

$$
\frac{\partial \alpha_{m}}{\partial t}+\nabla \cdot\left(U \alpha_{m}\right)+\nabla \cdot\left(U_{c} \alpha_{1}\right)=0
$$

Trong đó $U_{c}$ là thành phần vận tốc giao thoa giữa hai pha lỏng và khí.

Thuật toán PIMPLE áp dụng cho mô phỏng dòng chảy lũ bùn được minh họa ở hình 3.

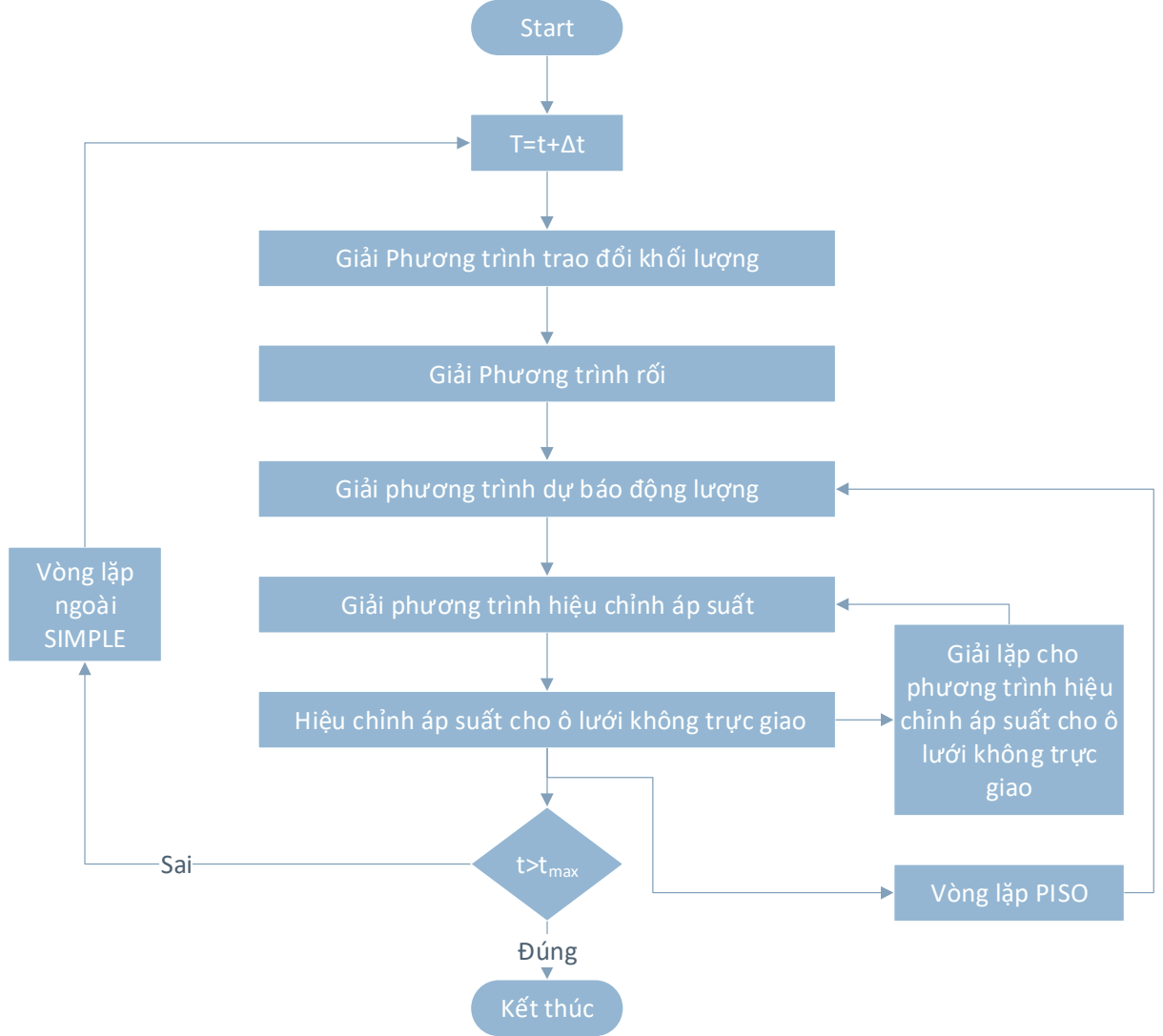

Hình 3. Thuật toán PIMPLE áp dụng cho mô phỏng lũ bùn đá.

\subsection{Các điều kiện đầu và biên}

Các dữ liệu thu thập phục vụ mô phỏng lũ bùn đá trong trường hợp nghiên cứu này gồm: thể tích dòng chảy, mật độ hỗn hợp $(\rho \mathrm{hh})$. Trong đó, thực nghiệm được tiến hành với tỷ lệ pha trộn giữa nước $\left(\rho_{\mathrm{w} / \mathrm{hh}}\right)$, bùn khoáng với kích thước mịn, bùn khoáng với kích thước thô, cát mịn, cát thô và các hạt có kích thước từ sỏi trở lên với góc ma sát $\delta=36^{\circ}$. Đối với pha khí, mật độ nước $\left(\rho_{\mathrm{w}}\right)$ và ứng suất mặt thoáng $\left(\tau_{00}\right)$ được miêu tả chi tiết ở Bảng 1 .

Bảng 1. Các tham số thiết lập và thu thập phục vụ mô phỏng dòng chảy lũ bùn.

\begin{tabular}{lccc}
\hline \multicolumn{1}{c}{ Tham số } & Ký hiệu & Giá trị & Đơn vị \\
\hline Mật độ nước & $\rho_{\mathrm{w}}$ & 1000 & $\mathrm{~kg} \cdot \mathrm{m}^{-3}$ \\
Mật độ hỗn hợp & $\rho_{\mathrm{hh}}$ & 1802 & $\mathrm{~kg} \cdot \mathrm{m}^{-3}$ \\
Úng suất mặt thoáng & $\tau_{00}$ & 41,33 & $\mathrm{~Pa}$ \\
Thể tích dòng chảy & $\mathrm{V}$ & 0,01 & $\mathrm{~m}^{3}$ \\
Tỷ lệ pha trộn nước & $\rho_{\mathrm{w} / \mathrm{hh}}$ & 28,5 & $\%$ \\
\hline
\end{tabular}




\begin{tabular}{lccc}
\hline \multicolumn{1}{c}{ Tham số } & Ký hiệu & Giá trị & Đơn vị \\
\hline Tỷ lệ bùn khoáng mịn & $\rho_{\text {sf } / h h}$ & 1,6 & $\%$ \\
Tỷ lệ bùn khoáng thô & $\rho_{\text {sr/hh }}$ & 8,8 & $\%$ \\
Tỷ lệ cát mịn & $\rho_{\text {sf } / h h}$ & 27,8 & $\%$ \\
Tỷ lệ cát thô & $\rho_{\text {sr/hh }}$ & 47,8 & $\%$ \\
Tỷ lệ hạt kích thước từ sỏi trở lên & $\rho_{\text {src/hh }}$ & 14,0 & $\%$ \\
\hline
\end{tabular}

Nghiên cứu được tiến hành với không gian lưới $\Delta \mathrm{x}=0,02 \mathrm{~m}, \Delta \mathrm{y}=0,01 \mathrm{~m}$ và $\Delta \mathrm{z}$ thay đổi từ 0,001 đến $0,025 \mathrm{~m}$ (Hình 4$)$. Để đảm bảo các thiết lập mô phỏng bắt được các chuyển động với vận tốc lớn, bước thời gian tính toán được thiết lập $\Delta \mathrm{t}=0,04$ giây.

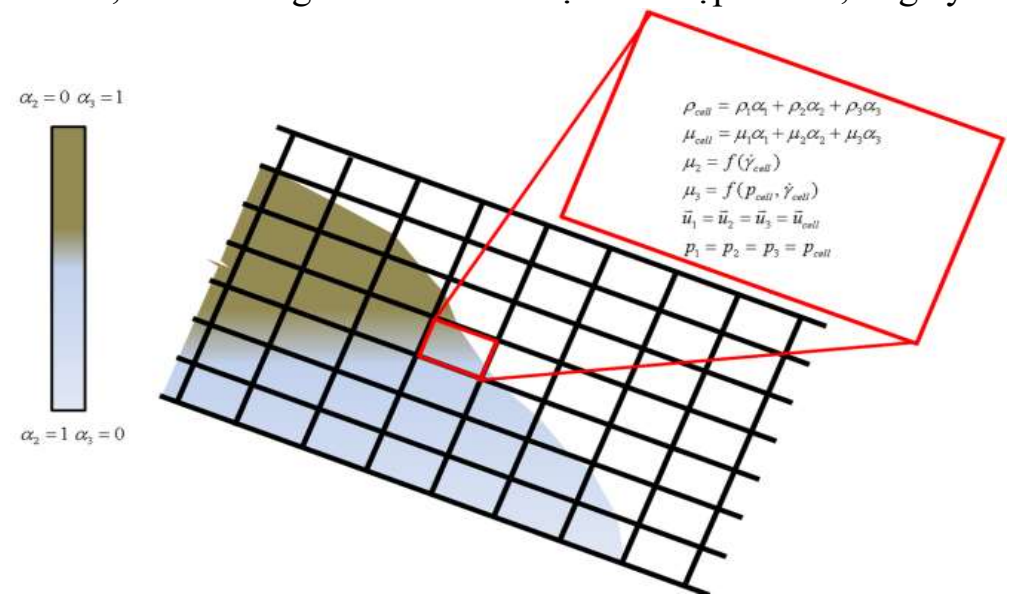

Hình 4. Minh họa lưới sử dụng mô phỏng dòng chảy lũ bùn đá [19].

\section{Kết quả và thảo luận}

\subsection{Kết quả mô phỏng dòng chảy lũ bùn}

Mô phỏng diễn biến dòng chảy hỗn hợp lũ bùn được minh họa trên hình 5 . Hình 5 thể hiện các khoảng thời gian di chuyển của dòng chảy lũ bùn từ lúc bắt đầu mô phỏng (Hình $5 \mathrm{a}$ ) đến khoảng thời gian sau 2 giây di chuyển của dòng lũ bùn (Hình $5 b$ ) và sau các khoảng thời gian di chuyển tiếp theo của chúng từ giây thứ tư (Hình $5 \mathrm{c}$ ), giây thứ 8,16 và giây thứ 20 (Hình $5 \mathrm{~d}, 5 \mathrm{e}$ và $5 \mathrm{f}$ ). Nhìn chung, hình 5 thể hiện kết quả mô phỏng dòng chảy lũ bùn từ phần mềm OpenFOAM phù hợp với xu thế diễn biến hiện tượng lũ bùn trong thực tế.

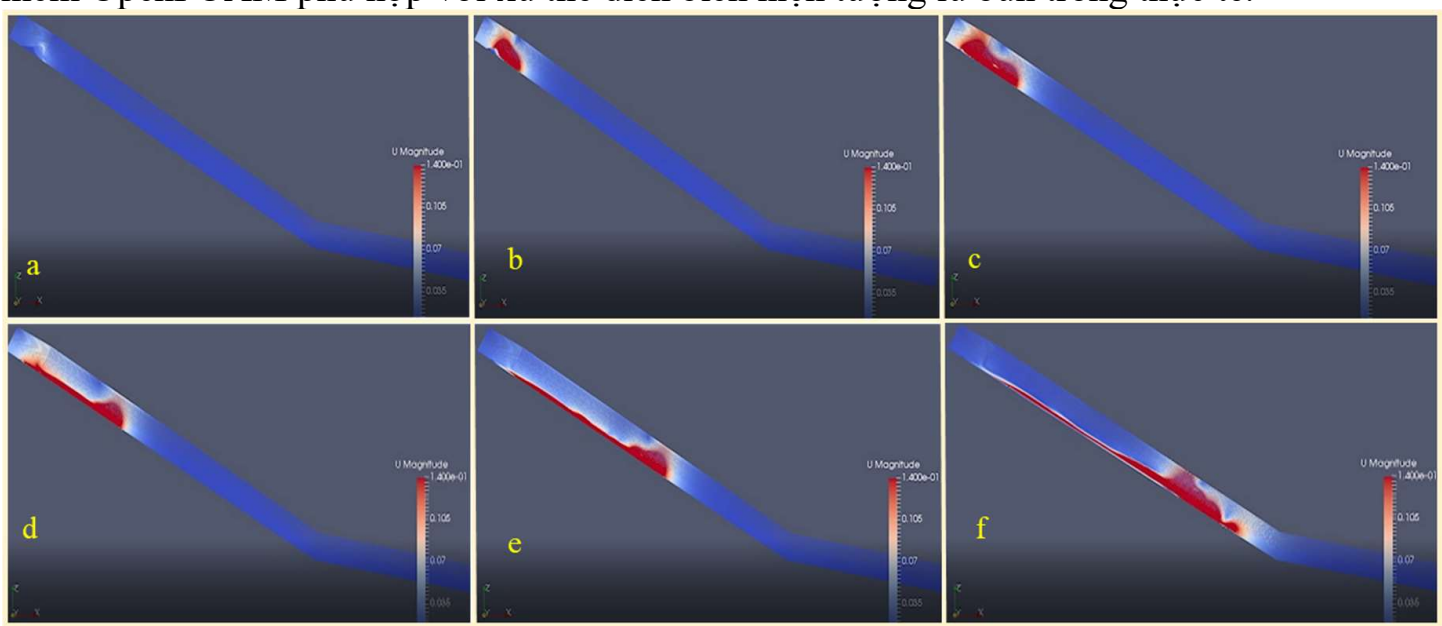

Hình 5. Minh họa kết quả mô phỏng dòng lũ bùn đá theo thời gian từ lúc bắt đầu xảy ra sự kiện (Hình $5 \mathrm{a}$ ) đến khi kết thúc sự kiện (Hình $5 \mathrm{f}$ ) của khối vật liệu nước và hỗn hợp bùn, đất và cát. 


\section{2. Đánh giá kết quả mô phỏng}

Kết quả mô phỏng dòng chảy lũ bùn từ phần mềm OpenFOAM được so sánh với dữ liệu đo đạc thực nghiệm và so với kết quả nghiên cứu từ công trình nghiên cứu đã được công bố [4] dùng phần mềm InterMixingflow cho cùng dữ liệu mà nghiên cứu này áp dụng (Bảng 2). Bảng 2 và hình $6 \mathrm{a}$ thể hiện dòng chảy lũ bùn mô phỏng từ phần mềm OpenFOAM cho trị số thấp hơn so với dữ liệu thực đo và phần mềm InterMixingflow. Tuy nhiên, về diễn biến xu thế và thời gian trễ của dòng chảy lũ bùn thì phần mềm OpenFOAM cho kết quả phù hợp hơn với dữ liệu thực đo và với kết quả mô phỏng từ phần mềm InterMixingflow (Hình 6b).
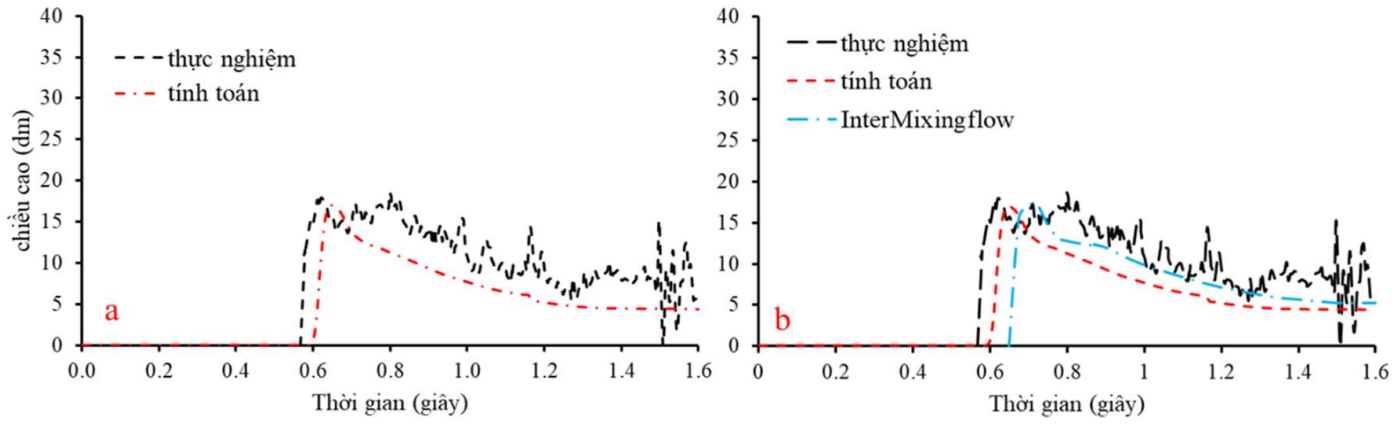

Hình 6. So sánh kết quả mô phỏng từ phần mềm OpenFOAM với a) dữ liệu đo đạc và b) với phần mềm InterMixingflow.

Bảng 2. Dữ liệu độ cao dòng chảy lũ bùn trích xuất từ dữ liệu đo đạc, mô phỏng từ phần mềm OpenFOAM và phần mềm InterMixingflow từ một nghiên cứu khác.

\begin{tabular}{|c|c|c|c|}
\hline \multirow{2}{*}{$\begin{array}{l}\text { Thời } \\
\text { gian }\end{array}$} & \multirow{2}{*}{$\begin{array}{l}\text { Dũ̃ liệu đo } \\
\text { đạc h (dm) }\end{array}$} & \multicolumn{2}{|c|}{ Mô phỏng từ các phần mềm $h(\mathrm{dm})$} \\
\hline & & OpenFOAM & InterMixingflow \\
\hline 0,60 & 15,02 & 0,56 & 0,00 \\
\hline 0,65 & 17,97 & 10,04 & 0,00 \\
\hline 0,70 & 14,28 & 15,47 & 15,59 \\
\hline 0,75 & 17,29 & 13,28 & 17,16 \\
\hline 0,80 & 14,91 & 12,16 & 14,85 \\
\hline 0,85 & 18,37 & 11,28 & 12,76 \\
\hline 0,90 & 13,44 & 10,36 & 12,34 \\
\hline 0,95 & 13,72 & 9,35 & 11,93 \\
\hline 1,00 & 12,76 & 8,29 & 10,67 \\
\hline 1,05 & 10,66 & 7,71 & 9,83 \\
\hline 1,10 & 12,87 & 7,04 & 9,10 \\
\hline 1,15 & 8,84 & 6,60 & 8,37 \\
\hline 1,20 & 10,20 & 6,15 & 7,74 \\
\hline 1,25 & 8,16 & 5,22 & 7,11 \\
\hline 1,30 & 6,35 & 4,85 & 6,69 \\
\hline 1,35 & 6,97 & 4,68 & 6,17 \\
\hline 1,40 & 8,79 & 4,56 & 5,86 \\
\hline 1,45 & 7,99 & 4,50 & 5,65 \\
\hline 1,50 & 8,84 & 4,46 & 5,44 \\
\hline 1,55 & 15,14 & 4,46 & 5,23 \\
\hline 1,60 & 1,76 & 4,46 & 5,23 \\
\hline 1,65 & 5,56 & 4,39 & 5,23 \\
\hline
\end{tabular}


So sánh kết quả tính toán dòng chảy lũ bùn từ phần mềm OpenFOAM và từ kết quả mô phỏng của [8] cho thấy, về trị số phần mềm OpenFOAM cho giá trị tính nhỏ hơn so với kết quả tính từ phần mềm InterMixingflow, tuy nhiên về xu hướng và thời gian trễ thì phần mềm OpenFOAM có thời gian trễ so với dữ liệu đo đạc nhỏ $(0,01$ giây) trong khi phần mềm InterMixingflow cho thời gian trễ ( 0,03 giây) (Bảng 2 , Hình $6 b)$.

\subsection{Phân tích mức độ phù hợp}

Mức độ phù hợp giữa kết quả mô phỏng diễn biến dòng chảy lũ bùn từ phần mềm OpenFOAM và phần mềm InterMixingflow được đánh giá thông qua các chỉ dẫn sai số tỷ lệ sai khác trung bình $\left(\mathrm{R}_{\text {mean }}\right)$ và sai số toàn phương trung bình $(\mathrm{RMSE})$. Bảng 3 thể hiện kết quả phân tích mức độ phù hợp của các phần mềm mô phỏng độ cao dòng chảy lũ bùn dựa

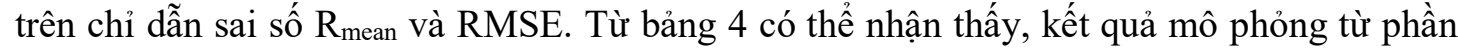

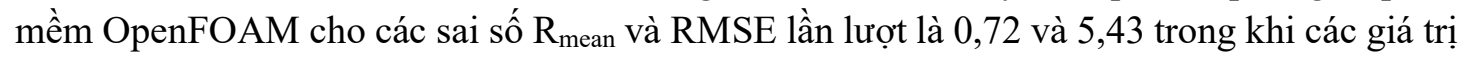
sai số tương ứng thu được từ phần mềm InterMixingflow lần lượt là 2.3 là 0,85 và 5,83 . Theo đó, chỉ số $\mathrm{R}_{\text {mean }}$ được xác định dựa trên so sánh kết quả mô phỏng của phần mềm OpenFOAM và dữ liệu đo đạc là 0,72 . Theo [21], khi chỉ số $\mathrm{R}_{\text {mean }}$ tiến đến 1,0 có nghĩa là kết quả tính toán của một yếu tố phù hợp tốt với thực tế và điều này đồng nghĩa với phương pháp được áp dụng cho một nghiên cứu bất kỳ có độ chính xác cao. Thêm vào đó, sai số RMSE tính được từ phần mềm OpenFOAM cho giá trị nhỏ hơn so với phần mềm InterMixingflow, điều đó có nghĩa rằng phần mềm OpenFOAM rất có triển vọng để xem xét áp dụng cho các nghiên cứu mô phỏng dòng chảy lũ bùn tương tự cho các kênh tự nhiên.

Bảng 3. Kết quả phân tích các chỉ số sai số thống kê cho các phần mềm mô phỏng.

\begin{tabular}{ccc}
\hline Phần mềm & $\mathrm{R}_{\text {mean }}$ & RMSE \\
\hline OpenFOAM & 0,72 & 5,43 \\
InterMixingflow & 0,85 & 5,83 \\
\hline
\end{tabular}

\subsection{Hạn chế của nghiên cứu}

Nghiên cứu được tiến hành dựa trên dữ liệu thực nghiệm mô hình vật lý, chính vì vậy một số tham số còn chưa được thu nhỏ quy mô phù hợp với thực tế (ví dụ như kích thước hạt sỏi, độ dốc kênh) và đây cũng chính là một trong những nguyên nhân dẫn đến kết quả mô phỏng còn số khác biệt so với thực tế.

\section{Kết luận}

Nghiên cứu được triển khai nhằm mục tiêu bước đầu thử nghiệm khả năng áp dụng phần mềm mã nguồn mở OpenFOAM mô phỏng dòng lũ bùn đá cho trường hợp kênh thực nghiệm. Kết quả thu được là cơ sở để xem xét áp dụng phần mềm OpenFOAM cho các nghiên cứu xa hơn đối với các kênh tự nhiên. Kết quả nghiên cứu cho thấy, phần mềm OpenFOAM bước đầu áp dụng mô phỏng độ cao dòng lũ bùn đá cho trường hợp kênh thực nghiệm thu được khá khả quan. Cụ thể, kết quả chạy mô phỏng độ cao dòng lũ bùn đá từ phần mềm mã nguồn mở OpenFOAM cho sai khác thông qua các chỉ số sai số thống kê tương đương với phần mềm InterMixingflow. Mặc dù kết quả nghiên cứu dòng lũ bùn đá thông qua áp dụng phần mềm OpenFOAM khả quan, tuy nhiên cần có những nghiên cứu xa hơn để đánh giá khả năng mô phỏng của phần mềm này cho những trường hợp sông, kênh trong thực tế.

Đóng góp của tác giả: Tác giả là người đã lên ý tưởng và tiến hành thực hiện nghiên cứu. 
Lời cảm ơn: Tác giả chân thành cảm ơn Giáo sư Paik Joongcheol-Viện nghiên cứu dòng chảy năng lượng cao, Đại học Quốc Gia Gangneung đã trao đổi, đóng góp ý kiến giúp tác giả hoàn thành nghiên cứu này.

Lời cam đoan: Tác giả cam đoan bài báo này là công trình nghiên cứu của tác giả, chưa được công bố ở đâu, không được sao chép từ những nghiên cứu trước đây; không có sự tranh chấp lợi ích.

\section{Tài liệu tham khảo}

1. Paik, J.C.; Park, S.D. Numerical simulation of flood and debris flow through drainage culvert. Proceeding of $5^{\text {th }}$ International Conference on Debris-Flow Hazards Mitigation: Mechanics, Prediction and Assessment 2011, 487-493.

2. Yu, B.; Ma, Y.; Xing Q. Experimental study on the influence of clay minerals on the yield stress of debris flows. J. Hydraul. Eng. 2013, 139, 364-373.

3. Takahashi, T. Debris flow, disaster prevention research institute, Kyoto University. Japan, Ann. Rev. Fluid Mech., 1981, 13, 57-77.

4. Albrecht, V.B. Debris inter-mixing-2.3: a finite volume for three-dimensional debris-flow simulations with two calibration parameters. Geosci. Model Dev. 2016, 9, 2909-2923.

5. Kiên, N.T. Nghiên cứu khả năng áp dụng đập hở khung thép ngăn lũ bùn đá tại khu vực miền núi phía bắc Việt Nam. Tạp chí Khoa học Công nghệ Xây dưng 2019 , 13(5), 28-37.

6. Yu, B.; Ma, Y.; Qi, X. Experimental study on the influence of clay minerals on the yield stress of debris flows. Eng. ASCE 2013, 139, 364-373.

7. Ko, S.M.; Lee, S.W.; Yune, C.Y.; Kim, G. Topographic analysis of landslides in Umyeonsan. J. Korean Society Sur. 2013, 32, 55-62.

8. Hürlimann, M.; McArdell, W.; Rickli, C. Field and laboratory analysis of the runout characteristics of hillslope debris flows in Switzerland. Geomorphology 2015, 232, 20-32. https://doi.org/10.1016/j.geomorph.2014.11.030.

9. Bull, J.M.; Miller, H.; Gravley, D.M. Assessing debris flow using LiDAR differencing: 18 May 2005 Matata event, New Zealand. Geomorphology, 2010, 124, $75-84$.

10. Logan, M.; Iverson, R. Video documentation of experiments at the USGS debrisflow flume 1992-2006. Website U.S. Geological Survey Open-File Report $2007-$ 1315, v.1.3, 2013. Available online: http://pubs.usgs.gov/of/2007/1315/ (last access: 5 February 2014).

11. Kim, H.; Lee, S.W.; Yune, C.Y.; Kim, G. Volume estimation of small-scale debris flows based on observations of topographic changes using airborne LiDAR DEMs. J. Mount. Sci. 2014, 11, 578-591.

12. Kim, G.; Yune, C.Y.; Paik, J.; Lee, S.W. Analysis of debris flow behavior using airborne lidar and image data. The International Archives of the Photogrammetry, Remote Sens. and Spatial Inf. Sci. Volume XLI-B8, 2016 XXIII ISPRS Congress, 12-19 July 2016, Prague, Czech Republic, 2016. 
13. Mergili, M.; Fischer, J.; Krenn, J.; Pudasaini, S.P. Rava-flow v1, an advanced opensource computational framework for the propagation and interaction of two-phase mass flows. Geosci. Model Dev. 2017, 10, 553-569.

14. Kattel, P.; Khattri, K.B.; Pokhrel, P.R.; Kafle, J.; Tuladhar, B.M.; Pudasaini, S.P. Simulating glacial lake outburst floods with a two-phase mass flow model. Ann. Glaciol. 2016, 57, 349-358.

15. King, H.M. What is a debris flow? Geoscience news and information. Tham khảo từ website: https://geology.com/articles/debris-flow/, 2018.

16. Lee, C.W.; Woo, C.S.; Youn, H.J. Analysis of debris flow hazard by the optimal parameters extraction of random walk model. J. Korean Forest Soc. 2011, 100, 664 671.

17. Scheidl, C.; Chiari, M.; Kaitna, R.; Müllegger, M.; Krawtschuk, A.; Zimmermann, T.; Proske, D. Analysing debris-flow impact models, based on a small-scale modelling approach. Surv Geophys. 2013, 34, 121-140.

18. Pudasaini, S. A general two-phase debris flow model. J. Geophys. Res. 2012, 117, F03010. https://doi.org/10.1029/2011JF002186.

19. OpenFOAM-Foundation: OpenFOAM Standard Solvers. Website User Guide of OpenFOAM, 2016, pp. 12. http://www.openfoam.org/docs/user/standardsovers.php.

20. O’Brian, J.; Julien, P.Y. Laboratory analysis of mudflow properties. J. Hydraul. Eng. 1988, 114, 877-887.

21. Bertolo, P.; Wieczorek, G.F. Calibration of numerical models for small debris flows in Yosemite Valley, California, USA. Nat. Hazards Earth Syst. Sci. 2005, 5, 993 1001. https://doi.org/10.5194/nhess-5-993-2005.

22. Wardle, K.E.; Weller, H. Hybrid multiphase CFD solver for coupled dispersed/segregated flows in liquid extraction. Int. J. Chem. Eng. 2013, 128936, https://doi.org/10.1155/2013/128936.

23. Yilma, H.; Moges, S.A. Application of semi-distributed conceptual hydrological model for flow forecasting on upland catchments of Blue Nile River Basin, a case study of Gilgel Abbay catchment. Catchment Lake Res. 2007, pp. 200. 


\title{
Application of the OpenFOAM open-source software debris flow simulations
}

Dang Truong An ${ }^{*}$

${ }^{1}$ University of Science, Vietnam National University, Ho Chi Minh City; dtan@hcmus.edu.vn

\begin{abstract}
Debris flow is a form of high-energy flow that includes a mixture of water, soil, sand and gravel and they frequently occur in mountainous areas. Debris flow generated a high-energy when they collide with obstacles in the flowing processes is enormous, which can result in massive loss of lives and property. In this study, the OpenFOAM software was initially tested to simulate the debris flow in the artificial channel. The performance of the applied software is assessed by comparing the simulated results with observed data and the simulated results of the InterMixingflow software. The statistical indicators of the OpenFOAM software are $\mathrm{R}_{\text {mean }}=0.72$ and $\mathrm{RMSE}=5.43$, respectively while the correspond values of the InterMixingflow software are $\mathrm{R}_{\text {mean }}=0.85$ and $\mathrm{RMSE}=5.83$, respectively. The obtained statistical indicators implied that the OpenFOAM software is capable to simulate the debris flow in the natural channel cases.
\end{abstract}

Keywords: Debris flow; OpenFOAM; High pressure; Typhoon; High energy flow. 